\title{
Cyclic Cohomology of Certain Affine Schemes
}

\author{
By
}

\author{
Tetsuya MASUDA* and Toshikazu NATSUME**
}

\begin{abstract}
Cyclic cohomology of the commutative $\mathbb{C}$-algebra $A=\mathbb{C}[x] /(f)$ associated with $f \in \mathbb{C}[x]$ is computed by making use of an explicitly constructed projective resolution. The result is $H^{\text {ovon }}(A) \cong \mathbb{C}^{m}$ and $H^{\text {odd }}(A) \cong 0$, where $m$ is the number of mutually distinct roots of $f=0$ in $\mathbb{C}$.
\end{abstract}

\section{\$1. Introduction}

Recently, cyclic cohomology of algebras was discovered by A. Connes in the formulation of non-commutative differential geometry [3], [4]. In connection with the pairing with algebraic or topological $K$-theory, cyclic cohomology is quite useful also for the study of $K$-theory. For instance, A. Connes uses cyclic cocycles to express certain characteristic classes of a foliation in connection with the topological $K$-theory of the associated foliation $C^{*}$-algebra, see [5]. In this context, it seems to be important to compute cyclic cohomology of interesting algebras, which appear in differential topology or in algebraic geometry.

In [6], cyclic cohomology of group algebras of free groups is computed. Dualizing cyclic cohomology, D. Quillen introduced cyclic homology of algebras [8]. In [1], cyclic homology of group rings of countable discrete groups with coefficient in commutative rings is computed in terms of classifying spaces and homotopy theory. It was shown in [4] that cyclic cohomology of $C^{\infty}(M)$ recovers the $\mathbb{C}$ -

Communicated by H. Araki, June 6, 1985.

* Mathematical Sciences Research Institute, Berkeley, California, 94720, USA.

Current address: Institute des Hautes Etudes Scientifiques, 35 route de Chartres, 91440 Bures-sur-Yvette, France.

** Mathematical Sciences Research Institute, Berkeley, California, 94720, USA and Department of Mathematics, Faculty of Science, Saitama University, Shimo-Okubo, Urawa, 338, Japan.

Research supported in part by NSF Grant 8120970 . 
coefficient de Rham homology of the compact smooth manifold $M$. Then it is natural to expect that cyclic cohomology of the $\boldsymbol{C}$-algebra $\boldsymbol{C}\left[x_{1}, \ldots, x_{n}\right]$ of polynomials in $n$-variable coincides with the de Rham homology of the affine spaces, and actually it does so, [7]. It now becomes an interesting problem to compute cyclic cohomology of the quotient algebra $\boldsymbol{C}\left[x_{1}, \ldots, x_{n}\right] / I$ by an ideal $I$.

The present paper is the first attempt in this direction. We compute cyclic cohomology of $\boldsymbol{C}$-algebras $A=\boldsymbol{C}[x] /(f), f \in \boldsymbol{C}[x]$. Our main result is:

Theorem. Let $m$ be the number of mutually distinct roots of $f=0$ for $f \in \boldsymbol{C}[x]$. Then $H^{\text {even }}(A) \cong \boldsymbol{C}^{m}$, and $H^{\text {odd }}(A)=0$.

Our proof is based on an explicit construction of projective resolution of $A$ as a module over the enveloping algebra $B$ of $A$, [2].

T. Goodwille seems to have first computed, but never published, the cyclic homology of $\boldsymbol{C}[x] /\left(x^{n+1}\right)$. His method is reported on by R. Staffeldt in [9]. Our computation is independent from the above.

Our research started during the participation of the second named author to Mathematical Sciences Research Institute project " $K$-theory, index theory and operator algebras."

The authors would like to thank Professor M. Takesaki for his valuable suggestions. The first named author is supported by the Educational Project for Japanese Mathematical Scientists and wishes to express his gratitude to the project.

\section{§2. Direct Sum Formula}

Let $A_{1}, A_{2}$ be $\boldsymbol{C}$-algebras, and let $A=A_{1} \oplus A_{2}$ be their direct sum. By the additivity theorem for Hochschild cohomology groups [Theorem 5. 3,2], we have a natural isomorphism

$$
H^{*}\left(A, A^{*}\right) \cong H^{*}\left(A_{1}, A_{1}^{*}\right) \oplus H^{*}\left(A_{2}, A_{2}^{*}\right) .
$$

By making use of a long exact sequence of $\mathrm{A}$. Connes relating cyclic cohomology to Hochschild cohomology [Theorem 37,4] together with the five lemma [Proposition 1.1,2] we have

$$
H_{\lambda}^{*}(A) \cong H_{\lambda}^{*}\left(A_{1}\right) \oplus H_{\lambda}^{*}\left(A_{2}\right) .
$$


Hence, the spectral sequence for $A$ splits into a direct sum and we obtain,

$$
\begin{aligned}
& H^{\text {even }}(A) \cong H^{\text {even }}\left(A_{1}\right) \oplus H^{\text {even }}\left(A_{2}\right), \\
& H^{\text {odd }}(A) \cong H^{\text {odd }}\left(A_{1}\right) \oplus H^{\text {odd }}\left(A_{2}\right) .
\end{aligned}
$$

Let $f \in \mathbb{C}[x]$. For the discussion of $\mathbb{C}[x] /(f)$, we can assume that $f$ is monic. Since our field $\boldsymbol{C}$ is algebraically closed, we can choose mutually distinct $\alpha_{1}, \ldots, \alpha_{m} \in \boldsymbol{C}$ such that

$$
f(x)=\left(x-\alpha_{1}\right)^{N_{1}} \cdots\left(x-\alpha_{m}\right)^{N_{m}},
$$

where $N_{1}, \ldots, N_{m}$ are positive integers satisfying $N_{1}+\ldots+N_{m}=\operatorname{deg}(f)$. Then, we have $\boldsymbol{C}$-algebra isomorphism

$$
\mathbb{C}[x] /(f) \cong \mathbb{C}[x] /\left(\left(x-\alpha_{1}\right)^{N_{1}}\right) \oplus \cdots \oplus \mathbb{C}[x] /\left(\left(x-\alpha_{m}\right)^{N_{m}}\right) .
$$

By the direct sum formula (2.2), it suffices to compute cyclic cohomology of each $\boldsymbol{C}[x] /\left(\left(x-\alpha_{j}\right)^{N_{j}}\right)$ to compute cyclic cohomology of $\mathbb{C}[x] /(f)$. We can also reduce the problem to the computation for $C[x] /\left(x^{n}\right), n \geq 1$. In the later sections, we compute cyclic cohomology of $\boldsymbol{C}[x] /\left(x^{n}\right), n \geq 2$.

Here, we introduce some notations. For each positive integer $n$, let $\Phi_{n}(x, y)$ denote the polynomial in two variables defined by

$$
\Phi_{n}(x, y)=x^{n-1}+x^{n-2} y+\cdots+x y^{n-2}+y^{n-1}
$$

for $n \geq 1$ and $\Phi_{0}(x, y)=0$. For any $m$-tuple $I=\left(i_{1}, \ldots, i_{m}\right)$ of integers, put $|I|=i_{1}+\cdots+i_{m}$. Throughout this paper, all tensor products are taken over the complex number field.

\section{§3. Projective Resolution}

From now on, let $A=\mathbb{C}[x] /\left(x^{n}\right), n \geq 2$. In this section, we construct a projective resolution of $A$ as a module over its enveloping algebra $B=A \otimes A^{o p p}$. Recall that $B$ is a $\mathbb{C}$-algebra generated by two linearly independent elements $x, y$ with the relations $x y=y x$ and $x^{n}=y^{n}=0$.

For $j \geq 0$, put $M_{j}=B$. Obviously, all $M_{j}$ 's are projective $B$-modules. Let $\varepsilon: M_{0} \rightarrow A$ be the canonical augmentation [p. 168,2], that is, $\varepsilon(x)=\varepsilon(y)=x$. Define $\partial_{2 j}: M_{2 j} \rightarrow M_{2 j-1}$ and $\partial_{2 j-1}: M_{2 j-1} \rightarrow M_{2 j-2}, j \geq 1$, by the multiplications by $\Phi_{n}(x, y)$ and $(x-y)$, respectively. Then we get a sequence of $B$-modules and $B$-homomorphisms: 


$$
0 \longleftarrow A \stackrel{\varepsilon}{\longleftarrow} M_{0} \stackrel{\partial_{1}}{\longleftarrow} M_{1} \stackrel{\partial_{2}}{\longleftarrow} M_{2} \longleftarrow \cdots
$$

It is obvious that the sequence (3.1) is a complex of $B$-modules.

Proposition 3. 1. The sequence (3.1) is exact.

Proof. Let $f \in$ ker $\varepsilon$. We may regard $f$ as a polynomial in two variables $x$ and $y$. Since $f \in \operatorname{ker} \varepsilon$, there exists $g \in \mathbb{C}[x]$ such that $f(x, x)=x^{n} g(x)$. This implies that $f(x, y)-x^{n} g(x)$ is divided by $(x-y)$. Thus, $f$ is contained in the image of $\partial_{1}$. This shows the exactness at $M_{0}$.

Let $f \in M_{2 j-1}, j \geq 1$, be such that $\partial_{2 j-1}(f)=0$. Then there exist $g_{1}, g_{2} \in \mathbb{C}[x, y]$ such that

$$
(x-y) f(x, y)=g_{1}(x, y) x^{n}+g_{2}(x, y) y^{n} .
$$

From this, it follows that

$$
g_{1}(x, x) x^{n}+g_{2}(x, x) x^{n}=0 .
$$

Since $C[x]$ is an integral domain, $g_{1}(x, x)+g_{2}(x, x)=0$, which says that

$$
g_{1}(x, y)+g_{2}(x, y)=(x-y) \tilde{g}(x, y)
$$

for some $\tilde{g} \in \boldsymbol{C}[x, y]$. Then

$$
(x-y) f(x, y)=(x-y) \tilde{g}(x, y) y^{n}+(x-y) \Phi_{n}(x, y) g_{1}(x, y) .
$$

From this, we have

$$
f(x, y)=\tilde{g}(x, y) y^{n}+\Phi_{n}(x, y) g_{1}(x, y) .
$$

Consequently, $f=\partial_{2 j}\left(g_{1}\right)$ in $M_{2 j-1}$. This shows the exactness at $M_{2 j-1}$.

Finally, let $f \in M_{2 j}, j \geq 1$, be an element of $\operatorname{ker}\left(\partial_{2 j}\right)$. Then $\Phi_{n}(x, y) f(x, y)=g_{1}(x, y) x^{n}+g_{2}(x, y) y^{n}$ for some $g_{1}, g_{2} \in \mathbb{C}[x, y]$. Let $w$ be a primitive $n$-th root of unity. Put $x=w^{j} y$ in the above equality to get

$$
g_{1}\left(w^{i} y, y\right)+g_{2}\left(w^{i} y, y\right)=0, \quad 1 \leq j \leq n-1 .
$$

This means that $g_{1}(x, y)+g_{2}(x, y)$ is divisible by $\left(x-w^{j} y\right), 1 \leq j \leq n-1$. Therefore $g_{1}(x, y)+g_{2}(x, y)$ is divisible by $\prod_{j=1}^{n-1}\left(x-w^{j} y\right)=\Phi_{n}(x, y)$. Hence, there exists $\tilde{g} \in \mathbb{C}[x, y]$ such that

$$
g_{1}(x, y)+g_{2}(x, y)=\Phi_{n}(x, y) \tilde{g}(x, y) .
$$

Then it is easy to see $f=\partial_{2 j+1}\left(-g_{2}\right)$, which shows the exactness at 
$M_{2 j}$.

Q.E.D.

\section{§4. Hochschild Cohomology}

The projective resolution (3.1) enables us to compute the Horchschild cohomology $H^{*}\left(A, A^{*}\right)$ of a $\mathbb{C}$-algebra $A$ with coefficient $A^{*}=$ $\operatorname{Hom}_{C}(A, \boldsymbol{C})$.

The cohomology $H^{*}\left(A, A^{*}\right)$ is isomorphic to that of the cochain complex

$$
0 \longrightarrow \operatorname{Hom}_{B}\left(M_{0}, A^{*}\right) \stackrel{d_{1}}{\longrightarrow} \operatorname{Hom}_{B}\left(M_{1}, A^{*}\right) \stackrel{d_{2}}{\longrightarrow} \cdots,
$$

where the map $d_{j}$ is the transpose of $\partial_{j}$.

Since $M_{j}=B, j \geq 0$, the above cochain complex turns out to be

$$
0 \longrightarrow A^{*} \stackrel{d_{1}}{\longrightarrow} A^{*} \stackrel{d_{2}}{\longrightarrow} A^{*} \longrightarrow \cdots \text {, }
$$

where $d_{2 j+1}=0$, and $\left(d_{2 j+2}(\varphi)\right)\left(x^{k}\right)=n \varphi\left(x^{n-1+k}\right)$ for $j \geq 0$. Hence we obtain,

$$
\begin{aligned}
& H^{0}\left(A, A^{*}\right) \cong \mathbb{C}^{n} \\
& H^{j}\left(A, A^{*}\right) \cong \mathbb{C}^{n-1}, \quad j \geq 1 .
\end{aligned}
$$

For each $0 \leq \mathrm{p} \leq n-1$, let $\delta^{(p)}$ denote the element of $A^{*}$ defined by

$$
\delta^{(p)}\left(a_{0}+a_{1} x+\cdots+a_{n-1} x^{n-1}\right)=a_{p^{\circ}}
$$

Each $\delta^{(p)}$ determines an element $\varphi_{p}$ of $\operatorname{Hom}_{B}\left(M_{j}, A^{*}\right)$ by the formula

$$
\varphi_{p}\left(1_{B}\right)=\delta^{(p)} \text {. }
$$

The coboundaries $d_{j}$ 's are given by the following formula

$$
\begin{aligned}
& d_{2 j-1}\left(\varphi_{p}\right)=0, \quad 0 \leq p \leq n-1, \\
& d_{2 j}\left(\varphi_{p}\right)=0, \quad 0 \leq p \leq n-2, \\
& d_{2 j}\left(\varphi_{n-1}\right)=n \varphi_{0}, \quad j \geq 1 .
\end{aligned}
$$

Hence, we can see that $H^{2 m}\left(A, A^{*}\right) \cong \mathbb{C}^{n-1}$ is spanned by the classes of $\varphi_{1}, \ldots, \varphi_{n-1} \in \operatorname{Hom}_{B}\left(M_{2 m}, A^{*}\right)$, and $H^{2 m-1}\left(A, A^{*}\right) \cong \mathbb{C}^{n-1}$ is spanned by the classes of $\varphi_{0}, \ldots, \varphi_{n-2} \in \operatorname{Hom}_{B}\left(M_{2 m-1}, A^{*}\right), m \geq 1$. We can also see that $H^{0}\left(A, A^{*}\right) \cong \mathbb{C}^{n}$ is spanned by $\varphi_{0}, \ldots, \varphi_{n-1} \in \operatorname{Hom}_{B}\left(M_{0}, A^{*}\right)$.

\section{Proposition 4. 1.}

(1) $H^{0}\left(A, A^{*}\right) \cong \mathbb{C}^{n}$ with its generators given by $\varphi_{0, \ldots, \varphi_{n-1} \in}$ $\operatorname{Hom}_{B}\left(M_{0}, A^{*}\right)$. 
(2) $H^{2 m}\left(A, A^{*}\right) \cong \boldsymbol{C}^{n-1}$ with its generators given by $\varphi_{1}, \ldots, \varphi_{n-1} \in$ $\operatorname{Hom}_{B}\left(M_{2 m}, A^{*}\right), m \geq 1$.

(3) $H^{2 m-1}\left(A, A^{*}\right) \cong \mathbb{C}^{n-1}$ with its generators given by $\varphi_{0}, \ldots, \varphi_{n-2} \in$ $\operatorname{Hom}_{B}\left(M_{2 m-1}, A^{*}\right), m \geq 1$.

\section{§5. Quasi-isomorphisms}

In this section, we give quasi-isomorphisms between our resolution (3.1) and the canonical projective resolution of $A$. This is used to compute the cyclic cohomology of $A$ in our later section.

To begin with, we give a brief description of the canonical projective resolution of $A$ as $B$-module.

Let $\tilde{M}_{j}=B \otimes\left[\bigotimes_{i=1}^{j} A\right]$. Then $\tilde{M}_{j}$ 's are projective left $B$-modules in a canonical manner. Define $b_{j}: \tilde{M}_{j} \rightarrow \tilde{M}_{j-1}, j \geq 1$, by the $B$-linear extension of

$$
\begin{aligned}
b_{j}\left(1_{\tilde{B}} \otimes a_{1} \otimes\right. & \left.\ldots \otimes a_{j}\right)=\left(a_{1} \otimes 1\right) \otimes a_{2} \otimes \cdots \otimes a_{j} \\
& +\sum_{l=1}^{j-1}(-1)^{l} 1_{B} \otimes a_{1} \otimes \cdots \otimes a_{l} a_{l+1} \otimes \cdots \otimes a_{j} \\
& +(-1)^{j}\left(1 \otimes a_{j}\right) \otimes a_{1} \otimes \cdots \otimes a_{j-1} .
\end{aligned}
$$

Then the sequence

$$
0 \longleftarrow A \stackrel{\varepsilon}{\longleftarrow} \tilde{M}_{0} \stackrel{b_{1}}{\longleftarrow} \tilde{M}_{1} \stackrel{b_{2}}{\longleftarrow} \cdots
$$

is a projective resolution of $A$ with explicitly given homotopy maps. We call (5.2) the canonical projective resolution.

Proposition 5. 1. There exists a family of B-module homomorphisms $h_{j}: M_{j} \rightarrow \tilde{M}_{j}, j \geq 0$, such that the following diagram is commutative:

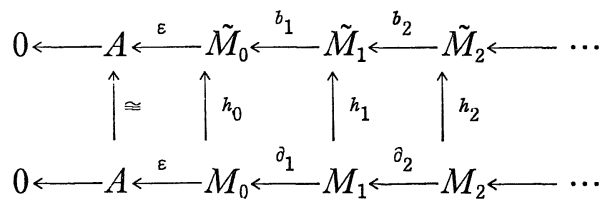

Proof. Since $M_{j}=B, j \geq 0$, it suffices to define $h_{j}$ for the unit $1_{B} \in M_{j}$. Put

$$
\begin{aligned}
h_{2 m}\left(1_{B}\right)=\sum_{1 \leqslant i_{1}, \cdots, i_{m} \leqslant n-1} x^{m(n-1)-\left(i_{1}+\cdots+i_{m}\right)} \otimes & \\
& \left(x \otimes x^{i_{1}} \otimes x \otimes x^{i_{2}} \otimes \cdots \otimes x \otimes x^{i_{m}}\right),
\end{aligned}
$$


Tetsuya Masuda and Toshikazu Natsume

1267

$(5.5)$

$$
\begin{aligned}
h_{2 m-1}\left(1_{B}\right)= & \sum_{1 \leqslant i_{1}, \cdots, i_{m-1} \leqslant n-1} x^{(m-1)(n-1)-\left(i_{1}+\cdots+i_{m-1}\right)} \otimes \\
& \left(x \otimes x^{i_{1}} \otimes x \otimes x^{i_{2}} \otimes \cdots \otimes x \otimes x^{i_{m-1}} \otimes x\right), \quad m \geq 1,
\end{aligned}
$$

and $h_{0}\left(1_{B}\right)=1_{B}$. By the definition, $\varepsilon \circ h_{0}=\varepsilon_{\circ}$. We show the commutativity of the diagram (5.3).

Let $l_{B} \in M_{2 m-1}$. We use an abbreviated notation by which we write $I=\left(i_{1}, \ldots, i_{m-1}\right)$ and we use $\sum_{I}$ instead of $\sum_{1 \leqslant i_{1}, \ldots, i_{m-1} \leqslant n-1}$. We compute

$$
\begin{array}{r}
b_{2 m-1}\left(h_{2 m-1}\left(1_{B}\right)\right)=\sum_{I} x^{(m-1)(n-1)-|I|} b_{2 m-1} \\
\left(1_{B} \otimes x \otimes x^{i_{1}} \otimes \cdots \otimes x \otimes x^{\left.i^{m-1} \otimes x\right) .}\right.
\end{array}
$$

We put

$$
\begin{aligned}
S_{1}= & \sum_{I} x^{(m-1)(n-1)-|I|+1} \otimes\left(x^{i_{1}} \otimes x \otimes x^{i_{2}} \otimes \cdots \otimes x \otimes x^{{ }^{i} m-1} \otimes x\right) \\
& -\sum_{I} x^{(m-1)(n-1)-|I|} \otimes\left(x^{i_{1}+1} \otimes x \otimes x^{i_{2}} \otimes \cdots \otimes x \otimes x^{i_{m-1}} \otimes x\right),
\end{aligned}
$$

$(5.8)$

$$
\begin{gathered}
S_{p}=\sum_{I} x^{(m-1)(n-1)-|I|} \otimes\left(x \otimes x^{i_{1}} \otimes \cdots \otimes x \otimes x^{i_{p-1}+1} \otimes x^{i_{p}} \otimes x \otimes \cdots\right. \\
\left.\otimes x^{i_{m-1}} \otimes x\right) \\
-\sum_{I} x^{(m-1)(n-1)-|I|} \otimes\left(x \otimes x^{i_{1}} \otimes \cdots \otimes x \otimes x^{i_{p-1}} \otimes x^{i_{p}+1} \otimes x \otimes \cdots\right. \\
\left.\otimes x^{i_{m-1}} \otimes x\right),
\end{gathered}
$$

for $2 \leq p \leq(m-1)$, and

$$
\begin{aligned}
S_{m}= & \sum_{I} x^{(m-1)(n-1)-|I|} \otimes\left(x \otimes x^{i_{1}} \otimes \cdots \otimes x \otimes x^{{ }^{i_{m-1}+1}}\right) \\
& -\sum x^{(m-1)(n-1)-|I|} y \otimes\left(x \otimes x^{i_{1}} \otimes \cdots \otimes x \otimes x^{i_{m-1}}\right) .
\end{aligned}
$$

Then, we obtain,

$$
b_{2 m-1} \circ h_{2 m-1}\left(1_{B}\right)=S_{1}+\cdots+S_{m} .
$$

It is also seen by (5.7) and (5.8), using $x^{n}=0$, that

$$
\begin{gathered}
S_{1}=\sum_{\substack{I \text { with } \\
i_{1}=1}} x^{(m-1)(n-1)-|I|+1} \otimes\left(x \otimes x \otimes x^{i_{2}} \otimes x \otimes \cdots \otimes x \otimes x^{i_{m-1}} \otimes x\right), \\
S_{p}=\sum_{\substack{I \text { with } \\
i_{p}=1}} x^{(m-1)(n-1)-|I|} \otimes\left(x \otimes x^{i_{1}} \otimes \cdots \otimes x \otimes x^{i_{p-1}+1} \otimes x \otimes x \otimes x^{i_{p+1}} \otimes\right. \\
\left.\cdots \otimes x^{i_{m-1}} \otimes x\right) \\
-\sum_{\substack{I \text { with } \\
i_{p-1}=1}} x^{(m-1)(n-1)-|I|} \otimes\left(x \otimes x^{i_{1}} \otimes \cdots \otimes x \otimes x^{i_{p}+1} \otimes x \otimes x^{i_{p+1}} \otimes\right. \\
\left.\cdots \otimes x^{i_{m-1}} \otimes x\right),
\end{gathered}
$$


1268

Cyclic Cohomology

for $2 \leq p \leq(m-1)$. So it follows that

$$
\begin{aligned}
& S_{1}+\cdots+S_{m-1}=x^{(m-1)(n-1)-m+1} \otimes(x \otimes \cdots \otimes x) \\
& +\sum_{\substack{I \text { with } \\
i_{2}=\cdots=i_{m-1}=1}} x^{(m-1)(n-1)-|I|} \otimes\left(x \otimes x^{i_{1}+1} \otimes x \otimes \cdots \otimes x\right)
\end{aligned}
$$

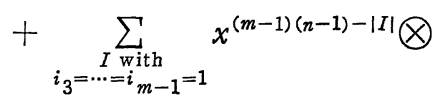

$$
\begin{aligned}
& \left(x \otimes x^{i_{1}} \otimes x \otimes x^{i_{2}+1} \otimes x \otimes \cdots \otimes x\right) \\
& +\cdots+\sum_{\substack{I \text { with } \\
i_{m-1}=1}} x^{(m-1)(n-1)-|I|} \otimes\left(x \otimes x^{i_{1}} \otimes x \otimes \cdots\right. \\
& \left.\otimes x \otimes x^{i_{m-2}+1} \otimes x \otimes x\right) \\
& =\sum_{1 \leqslant l_{1}, \cdots, l_{m-2} \leqslant n-1} x^{(m-1)(n-1)-\left(l_{1}+\cdots+l_{m-2}+1\right)+1} \otimes \\
& \left(x \otimes x^{l_{1}} \otimes \cdots \otimes x \otimes x^{l_{m-2}} \otimes x \otimes x\right) .
\end{aligned}
$$

Therefore, by putting $L=\left(p_{1}, \ldots, p_{m-1}\right)$,

$$
\begin{aligned}
S_{1}+\cdots+ & S_{m-1}+S_{m}=\sum_{L} x^{(m-1)(n-1)-|L|+1} \otimes\left(x \otimes x^{p_{1}} \otimes \cdots \otimes x \otimes x^{p_{m-1}}\right) \\
& -\sum_{L} x^{(m-1)(n-1)-|L|} \mid \mathcal{\otimes}\left(x \otimes x^{p_{1}} \otimes \cdots \otimes x \otimes x^{p_{m-1}}\right) \\
= & (x-y) \sum_{L} x^{(m-1)(n-1)-|L|} \otimes\left(x \otimes x^{p_{1}} \otimes \cdots \otimes x \otimes x^{p_{m-1}}\right) \\
= & (x-y) h_{2(m-1)}\left(1_{B}\right) \\
= & h_{2(m-1)} \circ \partial_{2 m-1}\left(1_{B}\right) .
\end{aligned}
$$

Thus, we obtain $b_{2 m-1} \circ h_{2 m-1}=h_{2(m-1)} \circ \partial_{2 m-1}$.

Suppose $1_{B} \in M_{2 m}$. We next compute

(5. 15) $\quad b_{2 m} \circ h_{2 m}\left(1_{B}\right)=b_{2 m}\left(\sum_{I} x^{m(n-1)-|I|} \otimes\left(x \otimes x^{i_{1}} \otimes \cdots \otimes x \otimes x^{i_{m}}\right)\right)$, with our abbreviated notation by $I=\left(i_{1}, \ldots, i_{m}\right)$. We put,

$$
\begin{aligned}
& T_{1}= \sum_{\substack{I \text { with } \\
i_{1}=1}} x^{m(n-1)-|I|+1} \otimes\left(x \otimes x \otimes x^{i_{2}} \otimes \cdots \otimes x \otimes x^{i_{m}}\right), \\
& T_{p}= \sum_{\substack{I \text { with } \\
i_{p}=1}} x^{m(n-1)-|I|} \otimes\left(x \otimes x^{i_{1}} \otimes \cdots\right. \\
& \quad-\sum_{\substack{I \text { with } \\
i_{p-1}=1}} x^{m(n-1)-|I|} \otimes\left(x \otimes x^{i_{p-1}+1} \otimes x \otimes x \otimes x^{i_{p+1}} \otimes \cdots x \otimes \cdots \otimes x^{i_{m}}\right) \\
&\left.\otimes x \otimes x^{i_{p}+1} \otimes x \otimes x^{i_{p+1}} \otimes \cdots \otimes x \otimes x^{i_{m}}\right),
\end{aligned}
$$


for $2 \leq p \leq M$. Then we obtain

$$
\begin{aligned}
& T_{1}+\cdots+T_{m}=x^{m(n-1)-m+1} \otimes(x \otimes \cdots \otimes x) \\
& +\sum_{i_{2}=\cdots=i_{m}=1} x^{m(n-1)-|I|} \otimes\left(x \otimes x^{i_{1}+1} \otimes x \otimes \cdots \otimes x\right) \\
& +\sum_{\substack{I \text { with } \\
i_{3}=\cdots=i_{m}=1}} x^{m(n-1)-|I|} \otimes\left(x \otimes x^{i_{1}} \otimes x \otimes x^{i_{2}+1} \otimes x \otimes \cdots \otimes x\right) \\
& +\cdots+\sum_{\substack{I \\
i_{m}=1}} x^{m(n-1)-|I|} \otimes\left(x \otimes x^{i_{1}} \otimes \cdots \otimes x \otimes x^{i_{m-1}+1} \otimes x\right) \\
& =x^{n-1} \sum_{\substack{\tilde{I}=\left(i_{1}, \cdots, i_{m-1}\right) \\
1 \leq i_{1}, \cdots, i_{m-1} \leq n-1}} x^{(m-1)(n-1)-|\tilde{I}|} \otimes\left(x \otimes x^{i_{1}} \otimes x \otimes \cdots \otimes x^{i_{m-1}} \otimes x\right) .
\end{aligned}
$$

Therefore,

$$
\begin{aligned}
b_{2 m} \circ h_{2 m}\left(1_{B}\right)= & T_{1}+\cdots+T_{m} \\
+ & \sum_{I} x^{m(n-1)+|I| y^{i}} \otimes\left(x \otimes x^{i_{1}} \otimes x \otimes x^{i_{2}} \otimes \cdots \otimes x \otimes x^{i_{m-1}} \otimes x\right) \\
= & \left(x^{n-1}+x^{n-2} y+\cdots+x y^{n-2}+y^{n-1}\right) \\
& \times \sum_{\substack{\tilde{I}=\left(i_{1}, \cdots, i_{m-1}\right) \\
1 \leq i_{1}, \cdots, i_{m-1} \leq n-1}} x^{(m-1)(n-1)-|\tilde{I}|} \otimes\left(x \otimes x^{i_{1}} \otimes \cdots \otimes x^{i_{m-1}} \otimes x\right) \\
= & \Phi_{n}(x, y) h_{2 m-1}\left(1_{B}\right) \\
= & h_{2 m-1} \circ \partial_{2 m}\left(1_{B}\right) .
\end{aligned}
$$

This completes the proof.

Q.E.D.

Proposition 5.2. There exists a family of B-module homomorphisms $k_{j}: \tilde{M}_{j} \rightarrow M_{j}, j \geq 0$, such that the following diagram is commutative:

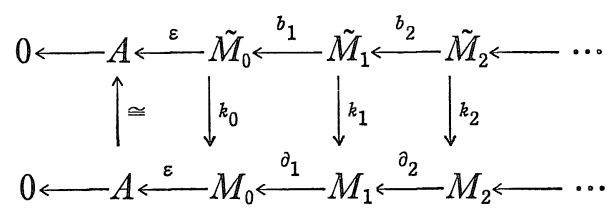

Proof. Put $k_{0}=$ identity of $B$, and define $k_{1}$ by

$$
k_{1}\left(1_{B} \otimes x^{i}\right)=\Phi_{i}(x, y) .
$$

For $m \geq 1$, put 
(5.22) $\quad k_{2 m}\left(1_{B} \otimes x^{i_{1}} \otimes x^{j_{1}} \otimes \cdots \otimes x^{i_{m}} \otimes x^{j_{m}}\right)$

$$
= \begin{cases}x^{i_{1}+j_{1}-n} \cdots x^{i_{m}+j_{m}-n} & \text { if } i_{l}+j_{l} \geq n \text { for } 1 \leq l \leq m \\ 0 & \text { else }\end{cases}
$$

and

$$
\begin{aligned}
& k_{2 m+1}\left(1_{B} \otimes x^{i_{1}} \otimes x^{j_{1}} \otimes \cdots \otimes x^{i_{m}} \otimes x^{j_{m}} \otimes x^{p}\right) \\
& \quad=k_{2 m}\left(1_{B} \otimes x^{i_{1}} \otimes x^{j_{1}} \otimes \cdots \otimes x^{i_{m}} \otimes x^{j_{m}}\right) \Phi_{p}(x, y) .
\end{aligned}
$$

Notice that, since $M_{2 m+1}=M_{2 m}=B,(5.23)$ has its meaning.

By the definition, $\varepsilon \circ k_{0}=\varepsilon$. Suppose $1_{B} \otimes x^{p} \in \tilde{M}_{1}$. Then

$$
\begin{aligned}
k_{0} \circ b_{1}\left(1_{B} \otimes x^{p}\right) & =x^{p}-y^{p} \\
& =(x-y) \Phi_{p}(x, y) \\
& =(x-y) k_{1}\left(1_{B} \otimes x^{p}\right) \\
& =\partial_{1} \circ k_{1}\left(1_{B} \otimes x^{p}\right) .
\end{aligned}
$$

Suppose $w=1_{B} \otimes x^{i_{1}} \otimes x^{j_{1}} \otimes \cdots \otimes x^{i_{m}} \otimes x^{j_{m}} \in \tilde{M}_{2 m} . \quad$ Then

$$
\begin{aligned}
b_{2 m}(w) & =x^{i_{1}} \otimes\left(x^{j_{1}} \otimes \cdots \otimes x^{i_{m}} \otimes x^{j_{m}}\right) \\
& -\sum_{l=1}^{m} 1_{B} \otimes x^{i_{1}} \otimes x^{j_{1}} \otimes \cdots \otimes x^{j_{l-1}} \otimes x^{i_{l}+j_{l}} \otimes x^{i_{l+1}} \otimes \cdots \otimes x^{i_{m}} \otimes x^{j_{m}} \\
& +\sum_{l=1}^{m-1} 1_{B} \otimes x^{i_{1}} \otimes x^{j_{1}} \otimes \cdots \otimes x^{i_{l}} \otimes x^{j_{l}+i_{l+1}} \otimes x^{j_{l+1}} \otimes \cdots \otimes x^{i_{m}} \otimes x^{j_{m}} \\
& +y^{j_{m}} \otimes\left(x^{i_{1}} \otimes x^{j_{1}} \otimes \cdots \otimes x^{i_{m-1}} \otimes x^{j_{m-1}} \otimes x^{i_{m}}\right) .
\end{aligned}
$$

We put,

$$
\begin{gathered}
S_{0}=k_{2 m-1}\left(x^{i_{1}} \otimes\left(x^{j_{1}} \otimes \cdots \otimes x^{i_{m}} \otimes x^{j_{m}}\right)\right), \\
\text { (5.27) } \quad S_{p}=k_{2 m-1}\left(1_{B} \otimes x^{i_{1}} \otimes x^{j_{1}} \otimes \cdots \otimes x^{i_{p}} \otimes x^{j_{p}+i_{p+1}} \otimes x^{j_{p+1}} \otimes \cdots \otimes x^{i_{m}} \otimes x^{j_{m}}\right), \\
1 \leq p \leq m-1,
\end{gathered}
$$

(5.28) $\quad S_{m}=k_{2 m-1}\left(y^{j_{m}} \otimes\left(x^{i_{1}} \otimes x^{j_{1}} \otimes \cdots \otimes x^{j_{m-1}} \otimes x^{i_{m}}\right)\right)$,

(5.29) $\quad T_{p}=-k_{2 m-1}\left(1_{B} \otimes x^{i_{1}} \otimes x^{j_{1}} \otimes \cdots \otimes x^{j_{p-1}} \otimes x^{i_{p}+j_{p}} \otimes x^{i_{p+1}} \otimes \cdots\right.$

$$
\left.\otimes x^{i} \otimes x^{j_{m}}\right), \quad 1 \leq p \leq m
$$

First, we assume $i_{1}+j_{1}<n$. Then by (5.22) and (5.23) we get

$$
k_{2 m-1} \circ b_{2 m}(w)=S_{0}+S_{1}+T_{1} \text {. }
$$

We have the following cases. 
Case (i). Assume $j_{1}+i_{2} \geq n$. Then $x^{j_{1}+i_{2}}=0$, and consequently,

$$
k_{2 m-1} \circ b_{2 m}(w)=S_{0}+T_{1}=0,
$$

because of the fact that $k_{2 m-1}\left(x^{i_{1}} \otimes\left(x^{j_{1}} \otimes \cdots \otimes x^{i_{m}} \otimes x^{j_{m}}\right)\right)$ is non zero if and only if $k_{2 m-1}\left(1_{B} \otimes x^{i_{1} j_{1}} \otimes x^{j_{2}} \otimes \cdots \otimes x^{j_{m}}\right)$ is non-zero, and if this is the case,

$$
S_{0}=x^{|I|+|\tilde{\jmath}|-(m-1) n} \Phi_{j_{m}}(x, y)=-T_{1},
$$

where $I=\left(i_{1}, \ldots, i_{m}\right)$ and $\tilde{J}=\left(j_{1}, \ldots, j_{m-1}\right)$.

Case (ii). Assume that $j_{1}+i_{2}<n$. Then

$$
k_{2 m-1} \circ b_{2 m}(w)=T_{1}+S_{1}=0 .
$$

Thus, if $i_{1}+j_{1}<n$, then $k_{2 m-1} \circ b_{2 m}(w)=0$. On the other hand, the condition $i_{1}+j_{1}<n$ implies that $k_{2 m}(w)=0$. Hence $k_{2 m-1} \circ b_{2 m}(w)=$ $\partial_{2 m} \circ k_{2 m}(w)$, if $i_{1}+j_{1}<n$.

We next assume $i_{1}+j_{1} \geq n$. We claim that if $i_{2}+j_{2}<n$, then $k_{2 m-1} \circ b_{2 n}(w)=0$. The conditions $i_{1}+j_{1} \geq n$ and $i_{2}+j_{2}<n$ imply

$$
k_{2 m-1} \circ b_{2 m}(w)=S_{0}+S_{1}+S_{2}+T_{2} \text {. }
$$

We have the following cases.

Case (1). Assume that $j_{1}+i_{2} \geq n, j_{2}+i_{3} \geq n$. Then $S_{1}=S_{2}=0$ and

$$
k_{2 m-1} \circ b_{2 m}(w)=S_{0}+T_{2}=0 \text {. }
$$

Case (2). Assume that $j_{1}+i_{2} \geq n, j_{2}+i_{3}<n$. Then $S_{0}=S_{1}=0$. Therefore

$$
k_{2 m-1} \circ b_{2 m}(w)=S_{2}+T_{2}=0 .
$$

Case (3). Assume that $j_{1}+i_{2}<n, j_{2}+i_{3} \geq n$. Then $S_{0}=S_{2}=0$. Hence

$$
k_{2 m-1} \circ b_{2 m}(w)=S_{1}+T_{2}=0 \text {. }
$$

Case (4). Assume that $j_{1}+i_{2}<n, j_{2}+i_{3}<n$. Then $S_{0}=S_{1}=0$. Consequently,

$$
k_{2 m-1} \circ b_{2 m}(w)=S_{2}+T_{2}=0 .
$$

On the other hand, if $i_{2}+j_{2}<n, k_{2 m}(w)=0$. This implies $k_{2 m-1} \mathrm{c} b_{2 m}(w)=$ $\partial_{2 m} \circ k_{2 m}(w)$ if $i_{2}+j_{2}<n$.

By the same argument as above, we can show that unless $i_{1}+j_{1} \geq$ 
$n, \ldots, i_{m}+j_{m} \geq n$, we obtain $k_{2 m-1} \circ b_{2 m}(w)=0$ and $k_{2 m}(w)=0$. So, in particular, $K_{2 m-1} \circ b_{2 m}(w)=\partial_{2 m} \circ k_{2 m}(w)$. Thus, we only have to show the commutativity of that diagram in the case when $i_{1}+j_{1} \geq n, \ldots, i_{m}+j_{m} \geq n$. Then $T_{1}=\ldots=T_{m-1}=0$ so that

$$
k_{2 m-1} \circ b_{2 m}(w)=S_{0}+S_{1}+\ldots+S_{m} .
$$

Put $I=\left(i_{1}, \ldots, i_{m}\right), J=\left(j_{1}, \ldots, j_{m}\right)$ and assume $|I|+|J|-m n \geq n$. Then

$$
k_{2 m}(w)=x^{|I|+|J|-m n}=0 .
$$

We put $\tilde{I}=\left(i_{1}, \ldots, i_{m-1}\right), \tilde{J}=\left(j_{1}, \ldots, j_{m-1}\right)$. Then we obtain

$$
\begin{aligned}
& S_{m}=y^{j_{m}} \Phi_{i_{m}}(x, y) x^{|\tilde{I}|+\mid \tilde{J}^{-(m-1) n}} y^{j_{m}}\left\{x^{i_{m}-1}+x^{i_{m}-2} y+\cdots\right. \\
& \left.+x^{i_{m}+j_{m}-n} y^{n-1-j_{m}}\right\} \times x^{|\tilde{I}|+\left|\jmath^{\jmath}\right|-(m-1) n} \\
& =0
\end{aligned}
$$

due to $i_{m}+j_{m}-n+|\tilde{I}|+|\tilde{J}|-(m-1) n=|I|+|J|-m n \geq n$. We next claim $S_{0}=0$. Unless $j_{1}+i_{2} \geq n, \ldots, j_{m-1}+i_{m} \geq n, S_{0}=0$. Suppose that $j_{1}+i_{2} \geq n, \ldots, j_{m-1}+i_{m} \geq n$. We put

$$
r=\left(j_{1}+i_{2}-n\right)+\cdots+\left(j_{m-1}+i_{m}-n\right) .
$$

If $r \geq n$, then we see

$$
S_{0}=k_{2 m-1}\left(x^{i_{1}} \otimes\left(x^{j_{1}} \otimes \cdots \otimes x^{i_{m}} \otimes x^{j_{m}}\right)\right)=0 .
$$

Suppose $r \leq n-1$. Then $r+i_{1}+j_{m}-n \geq n$, which implies $i_{1}+j_{m} \geq 2 n-r$. Therefore,

$$
i_{1} \geq 2 n-r-j_{m} \geq 2 n-r-(n-1)=n-r+1 \text {. }
$$

Hence,

$$
\begin{aligned}
S_{0} & =k_{2 m-1}\left(x^{i_{1}} \otimes\left(x^{j_{1}} \otimes \cdots \otimes x^{i_{m}} \otimes x^{j_{m}}\right)\right) \\
& =x^{i_{1}} x^{r} \Phi_{j_{m}}(x, y)=0
\end{aligned}
$$

due to $i_{1}+r \geq n+1$. Thus, if $|I|+|J|-m n \geq n, S_{0}=0$.

Next, suppose $S_{p} \neq 0$ for some $1 \leq p \leq m-1$. Then $i_{1}+j_{1} \geq n, \ldots$, $i_{p-1}+j_{p-1} \geq n, \quad i_{p}+j_{p}+i_{p+1} \geq n, \quad j_{p+1}+i_{p+2} \geq n, \ldots, j_{m-1}+i_{m} \geq n, \quad j_{p}+i_{p+1}<n$, and $\tilde{r}=\left(i_{1}+j_{1}-n\right)+\cdots+\left(i_{p}+j_{p}+i_{p+1}-n\right)+\cdots+\left(j_{m-1}+i_{m}-n\right)<n$. This implies $j_{m} \geq 2 n-\tilde{r}>n$ and hence, this is a contradiction. Therefore $S_{p}=0$ for $1 \leq p \leq m-1$. This implies that if $|I|+|J|-m n \geq n$, we obtain $k_{2 m-1} \circ b_{2 m}(w)=\partial_{2 m} \circ k_{2 m}(w)=0$. 
Assume that $p=|I|+|J|-m n<n$. Then $k_{2 m}(w)=x^{p}$. Therefore,

$$
\begin{aligned}
\partial_{2 m} \circ k_{2 m}(w) & =x^{p} \Phi_{n}(x, y) \\
& =x^{p}\left\{x^{n-p-1} y^{p}+x^{n-p-2} y^{p+1}+\cdots+x y^{n-2}+y^{n-1}\right\} .
\end{aligned}
$$

We also obtain

$$
\begin{aligned}
S_{m} & =y^{j_{m}} x^{q} \Phi_{i_{m}}(x, y) \\
& =y^{j_{m}} x^{q}\left\{x^{i^{-1}}+x^{i_{m^{-2}}} y+\cdots+x^{i_{m}+j_{m}-n} y^{n-1-j_{m}}\right\},
\end{aligned}
$$

where $q=|\tilde{I}|+|J|-(m-1) n$.

We further assume $p \geq i_{m}$. Then,

$$
\begin{aligned}
S_{m} & =y^{j_{m}} x^{q}\left\{x^{i_{m}-1+j_{m}-p} y^{p-j_{m}}+\cdots+x^{i_{m}+j_{m}-n} y^{n-1-j_{m}}\right\} \\
& =x^{n-1} y^{p}+x^{n-2} y^{p+1}+\cdots+x^{p+1} y^{n-2}+x^{p} y^{n-1} .
\end{aligned}
$$

If $S_{0} \neq 0$, then $j_{1}+i_{2} \geq n, \ldots, j_{m-1}+i_{m} \geq n$, and

$$
S_{0}=x^{i_{1}}\left\{x^{\left(j_{1}+i_{2}-n\right)+\cdots+\left(j_{m-1}+i_{m}-n\right)}\right\} \Phi_{j_{m}}(x, y) \text { 。 }
$$

By using

$$
\begin{aligned}
\left(j_{1}+i_{2}-n\right)+\cdots+\left(j_{m-1}+i_{m}-n\right) & =p-i_{1}+\left(n-j_{m}\right) \\
& =\left(p-j_{m}\right)+n-i_{1}
\end{aligned}
$$

we have

$$
S_{0}=x^{n+\left(p-j_{m}\right)} \Phi_{j_{m}}(x, y)=0
$$

due to $p-j_{m} \geq 0$. This contradicts to the assumption $S_{0} \neq 0$. Hence, $S_{0}=0$. Suppose $S_{l} \neq 0$ for some $1 \leq l \leq m-1$, then $S_{l}$ must be of the form

$$
\begin{aligned}
S_{l} & =x^{\left(i_{1}+j_{1}-n\right)+\cdots+\left(i_{l-1}+j_{l-1}-n\right)+\left(i_{l}+j_{l}+i_{l+1}-n\right)+\left(j_{l+1}+i_{l+2}-n\right)+\cdots+\left(j_{m-1}+i_{m}-n\right)} \\
& \Phi_{j_{m}}(x, y) \\
& =x^{|\tilde{I}|+|\tilde{J}|-(m-1) n_{m}} \Phi_{j_{m}}(x, y) \\
& =0
\end{aligned}
$$

which contradicts the assumption $S_{l} \neq 0$. Therefore $S_{l}=0$ for $1 \leq l \leq m-1$. Thus, by using (5.46) and (5.48), we obtain $k_{2 m-1} \circ b_{2 m}(w)=\partial_{2 m} \circ k_{2 m}(w)$ for $j_{m} \leq p<n$.

Finally, we assume $p<i_{m}<n$. In this case, (5.47) is equal to 


$$
S_{m}=y^{j_{m}} x^{n-j_{m}+p-1}+y^{j_{m}+1} x^{n-j_{m}+p-2}+\cdots+x^{i_{m}+j_{m}-n} y^{n-1} .
$$

It is then seen that exactly one of the following cases occurs:

$$
j_{1}+i_{2} \geq n, \ldots, j_{m-1}+i_{m} \geq n,
$$

$$
j_{1}+i_{2}<n, j_{2}+i_{3} \geq n, \ldots, j_{m-1}+i_{m} \geq n \text {, }
$$

$$
j_{l-1}+i_{l}<n, j_{l}+i_{l+1} \geq n, \ldots, j_{m-1}+i_{m} \geq n
$$

(c. $(m-1)) \quad j_{m-1}+i_{m}<n$.

Assume (c. 1). Then $S_{1}=\ldots=S_{m-1}=0$ and

$$
\begin{aligned}
S_{0} & =x^{i_{1}+\left(j_{1}+i_{2}-n\right)+\cdots+\left(j_{m-1}+i_{m}-n\right)} \Phi_{j_{m}}(x, y) \\
& =x^{n-\left(j_{m}-p\right)}\left\{x^{j_{m}-1}+x^{j_{m}-2} y+\cdots+x y^{j_{m}-2}+y^{j_{m}-1}\right\} \\
& =x^{n-1} y^{p}+x^{n-2} y^{p+1}+\cdots+x^{n-\left(j_{m}-p\right)} y^{j_{m}-1} .
\end{aligned}
$$

Therefore

(5. 55) $\quad k_{2 m-1} \circ b_{2 m}(w)=x^{n-1} y^{p}+x^{n-2} y^{p+1}+\cdots+x^{p} y^{n-1}=\partial_{2 m} \circ k_{2 m}(w)$.

Assume (c. $l$ ) for some $2 \leq l \leq m-1$. Then, $S_{0}=\cdots=S_{l-1}=S_{l+1}=\cdots$ $=S_{m-1}=0$, and

$$
S_{1}=x^{i} \Phi_{j_{m}}(x, y)
$$

where

$$
\text { (5.57) } \begin{aligned}
\tilde{l} & =\left(i_{1}+j_{1}-n\right)+\cdots+\left(i_{l-1}+j_{l-1}-n\right)+\left(i_{l}+j_{l}+i_{l+1}-n\right)+\cdots \\
& +\left(j_{m-1}+i_{m}-n\right) \\
& =|\tilde{I}|+|J|-(m-1) n+i_{m} \\
& =p-j_{m}+n .
\end{aligned}
$$

Therefore

$$
S_{1}=x^{n-1} y^{p}+x^{n-2} y^{p+1}+\cdots+x^{n-\left(j_{m}-p\right)} y^{j_{m}-1}
$$

So, we have

$$
k_{2 m-1} \circ b_{2 m}(w)=S_{l}+S_{m}=x^{n-1} y^{p}+x^{n-2} y^{p+1}+\cdots+x^{p} y^{n-1} .
$$

Thus we obtain $k_{2 m-1} \circ b_{2 m}(s)=\partial_{2 m} \circ k_{2 m}$ in all cases.

By similar, but tedious arguments, the equality $k_{2 m} \circ b_{2 m+1}=$ $\partial_{2 m+1} \circ k_{2 m+1}$ is shown. $\quad$ Q. E. D. 
Remark. By making use of the explicit description of $h_{n}$ and $k_{n}$, $n \geq 1$, we can show that $k_{n} \circ h_{n}=1$ on $M_{n}$. This, together with the commutativity of the diagrams by Proposition 5.1 and 5.2, gives us homotopy maps of our resolution (3.1) using the explicitly given homotopy maps of the canonical projective resolution (5.2). This means that our resolution (3.1) is a retraction of the canonical resolution (5.2).

\section{§6. Computation of Cyclic Cohomology}

In this section, we compute cyclic cohomology of $A=\mathbb{C}[x] /\left(x^{n}\right)$. Our computation is based on the spectral sequence associated with the exact couple of A. Connes, see [4].

We compute the total differential

$$
D_{n}: H^{n}\left(A, A^{*}\right) \rightarrow H^{n-1}\left(A, A^{*}\right), \quad n \geq 1,
$$

in terms of the basis obtained in Section 4 using the quasi-isomorphisms given in Section 5.

We first compute $D_{2 m+1}: H^{2 m+1}\left(A, A^{*}\right) \rightarrow H^{2 m}\left(A, A^{*}\right)$. Recall that $H^{2 m+1}\left(A, A^{*}\right)$ is spanned by the following cochains

$$
\begin{array}{r}
\phi_{j}\left(a_{0}, \ldots, a_{2 m+1}\right)=\varphi_{j}\left(k_{2 m+1}\left(1_{B} \otimes a_{1} \otimes \ldots \otimes a_{2 m+1}\right)\right)\left(a_{0}\right), \\
0 \leq j \leq n-2 .
\end{array}
$$

It suffices to compute

$$
\Xi=\left(h_{2 m}^{*} \tilde{B} k_{2 m+1}^{*} \varphi_{j}\right)(a), \quad a \in A,
$$

where $\tilde{B}$ is the map on the cochain level which induces the total differential $D_{2 m+1}$, see [4]. We have

$$
\begin{aligned}
\Xi= & \left(\tilde{B} k_{2 m+1}^{*} \varphi_{j}\right)\left(\sum_{I} x^{m(n-1)-|I|} \otimes\left(x \otimes x^{i_{1}} \otimes x \otimes \cdots \otimes x \otimes x^{i_{m}}\right)\right)(a) \\
= & \sum_{I}\left(\tilde{B} \psi_{j}\right)\left(x^{m(n-1)-|I|} a, x, x^{i_{1}}, x, \ldots, x, x^{i_{m}}\right) \\
= & \sum_{I}\left\{\left(B_{0} \psi_{j}\right)\left(x^{m(n-1)-|I|} a, x, x^{i_{1}}, x, \ldots, x, x^{i_{m}}\right)\right. \\
& +\left(B_{0} \psi_{j}\right)\left(x^{i_{m}}, x^{m(n-1)-|I|} a, x, x^{i_{1}}, \ldots, x^{i_{m}-1}, x\right) \\
& \left.+\ldots+\left(B_{0} \psi_{j}\right)\left(x, x^{i_{1}}, x, x^{i_{2}}, \ldots, x, x^{i_{m}}, x^{m(n-1)-|I|} a\right)\right\} \\
= & \sum_{I}\left\{\psi_{j}\left(1, x^{m(n-1)-|I|} a, x, x^{i_{1}}, x, \ldots, x, x^{i_{m}}\right)\right. \\
& -\psi_{j}\left(x^{m(n-1)-|I|} a, x, x^{i_{1}}, x, \ldots, x, x^{i_{m}}, 1\right)
\end{aligned}
$$


1276

Cyclic Cohomology

$$
\begin{aligned}
& +\cdots+\varphi_{j}\left(1, x, x^{i_{1}}, \ldots, x, x^{i_{m}}, x^{m(n-1)-|I|} a\right) \\
& \left.-\psi_{j}\left(x, x^{i_{1}}, \ldots, x, x^{i_{m}}, x^{m(n-1)-|I|} a, 1\right)\right\} \\
= & \sum_{I}\left\{\varphi_{j}\left(k_{2 m+1}\left(1_{B} \otimes x^{m(n-1)-|I|} a \otimes x \otimes x^{i_{1}} \otimes \cdots \otimes x^{i_{m}}\right)\right)(1)\right. \\
& -\varphi_{j}\left(k_{2 m+1}\left(1_{B} \otimes x \otimes x^{i_{1}} \otimes \cdots \otimes x \otimes x^{i_{m}} \otimes 1\right)\right)\left(x^{m(n-1)-|I|} a\right) \\
& +\varphi_{j}\left(k_{2 m+1}\left(1_{B} \otimes x^{m(n-1)-|I|} a \otimes x \otimes x^{i_{1}} \otimes \cdots \otimes x\right)\right)(1) \\
& -\varphi_{j}\left(k_{2 m+1}\left(1_{B} \otimes x^{m(n-1)-|I|} a \otimes x \otimes x^{i_{1}} \otimes \cdots \otimes x \otimes 1\right)\right)\left(x^{i_{m}}\right) \\
& +\cdots+\varphi_{j}\left(k_{2 m+1}\left(1_{B} \otimes x \otimes x^{i_{1}} \otimes \cdots \otimes x^{i_{m}} \otimes x^{m(n-1)-|I|} a\right)\right)(1) \\
& \left.-\varphi_{j}\left(k_{2 m+1}\left(1_{B} \otimes x^{i_{1}} \otimes x \otimes \cdots \otimes x^{m(n-1)-|I|} a \otimes 1\right)\right)(x)\right\} \\
= & \sum_{I}\left\{\varphi_{j}\left(k_{2 m+1}\left(1_{B} \otimes x^{m(n-1)-|I|} a \otimes x \otimes x^{i_{1}} \otimes \cdots \otimes x^{i_{m}}\right)\right)(1)\right. \\
& +\cdots+\varphi_{j}\left(k_{2 m+1}\left(1_{B} \otimes x \otimes x^{i_{1}} \otimes \cdots \otimes x^{m(n-1)-|I|} a\right)\right)(1) .
\end{aligned}
$$

Put

(6. 5) $\quad \Xi_{l}=\sum_{I} \varphi_{j}\left(k_{2 m+1}\left(1_{B} \otimes x^{i^{i}} \otimes x \otimes \cdots \otimes x^{i_{m}} \otimes x^{m(n-1)-|I|} \mathrm{a} \otimes x \otimes x^{i_{1}} \otimes \cdots\right.\right.$

$\left.\left.\otimes x^{i^{i}-1} \otimes x\right)\right)(1)$

for $1 \leq l \leq m$,

(6. 6) $\quad \Pi_{0}=\sum_{I} \varphi_{j}\left(k_{2 m+1}\left(1_{B} \otimes x^{m(n-1)-|I|} a \otimes x \otimes x^{i_{1}} \otimes \cdots \otimes x^{i_{m}}\right)\right)(1)$,

(6. 7) $\quad \Pi_{l}=\sum_{I} \varphi_{j}\left(k_{2 m+1}\left(1_{B} \otimes x \otimes x^{i_{m-l+1}} \otimes x \otimes \cdots \otimes x^{i_{m}} \otimes x^{m(n-1)-|I|} \otimes x \otimes\right.\right.$

$$
\left.\left.x^{i_{1}} \otimes \cdots \otimes x^{i_{m-l}}\right)\right)(1),
$$

for $1 \leq l \leq m-1$, and

(6. 8) $\quad \Pi_{m}=\sum_{I} \varphi_{j}\left(k_{2 m+1}\left(1_{B} \otimes x \otimes x^{i_{1}} \otimes \cdots \otimes x^{i_{m}} \otimes x^{m(n-1)-|I|} a\right)\right)(1)$.

We now put $a=x^{p}, 1 \leq p \leq n-1$. Unless $\left(i_{1}, \ldots, i_{m}\right)=(n-1, \ldots$, $n-1, p)$, we have

$$
k_{2 m+1}\left(1_{B} \otimes x^{m(n-1)-|I|} a \otimes x \otimes x^{i_{1}} \otimes \cdots \otimes x^{i_{m}}\right)=0 .
$$

Therefore,

$$
\begin{aligned}
\Pi_{0} & =\varphi_{j}\left(\Phi_{p}(x, y)\right)(1) \\
& =\delta^{(j)}\left(p x^{p-1}\right) \\
& = \begin{cases}j+1 & \text { if } p=j+1 \\
0 & \text { else. }\end{cases}
\end{aligned}
$$


Similarly,

$$
\Pi_{1}= \begin{cases}j+1 & \text { if } p=j+1 \\ 0 & \text { else }\end{cases}
$$

for $1 \leq l \leq m$. On the other hand, unless $\left(i_{1, \ldots,}, i_{m-1}\right)=(n-1, \ldots$, $n-1)$, we have

(6. 12) $\quad k_{2 m+1}\left(1_{B} \otimes x^{i_{l}} \otimes x \otimes \cdots \otimes x^{i_{m}} \otimes x^{m(n-1)-|I|+p} \otimes x \otimes x^{i_{1}} \otimes \cdots \otimes x^{i_{l}-1} \otimes x\right)$

$$
=0, \quad 1 \leq l \leq m \text {. }
$$

Therefore

$$
\begin{aligned}
\Xi_{l}= & \sum_{1 \leq i_{m} \leq n-1} \varphi_{j}\left(k _ { 2 m + 1 } \left(1_{B} \otimes x^{n-1} \otimes x \otimes x^{n-1} \otimes \cdots\right.\right. \\
& \left.\left.\cdots \otimes x^{i} m \otimes x^{m(n-1)-|I|+p} \otimes x \otimes x^{n-1} \otimes \cdots \otimes x\right)\right)(1) \\
= & \sum_{1 \leq i_{m} \leq n-1} \varphi_{j}\left(x^{p-1}\right)(1) \\
= & (n-1) \delta^{(j)}\left(x^{p-1}\right) \\
= & \begin{cases}n-1 & \text { if } p=j+1 \\
0 & \text { else. }\end{cases}
\end{aligned}
$$

Thus, we get

$$
D_{2 m+1}\left(\delta^{(j)}\right)=\{m(n-1)+(m+1)(j+1)\} \delta^{(j+1)}, \quad m \geq 1 .
$$

It is also seen that (6.14) holds also for $m=0$. This implies that

$$
D_{2 m+1}: H^{2 m+1}\left(A, A^{*}\right) \rightarrow H^{2 m}\left(A, A^{*}\right), \quad m \geq 1
$$

is isomorphic, and

$$
D_{1}: H^{1}\left(A, A^{*}\right) \rightarrow H^{0}\left(A, A^{*}\right)
$$

is injective with 1 -dimensional cokernel spanned by $\delta^{(0)}$. Hence, by using $D_{2 m} D_{2 m+1}=0, \quad m \geq 1$,

$$
D_{2 m}: H^{2 m}\left(A, A^{*}\right) \rightarrow H^{2 m-1}\left(A, A^{*}\right), \quad m \geq 1,
$$

is a zero map. Therefore, the chain complex $\left(H^{*}\left(A, A^{*}\right), D_{*}\right)$ is acyclic except on $H^{0}\left(A, A^{*}\right)$, and we obtain $E_{1}^{j}(A)=0$ for $j \geq 1$ and $E_{1}^{0}(A) \cong \mathbb{C}$ with its generator given by $\delta^{(0)}$. We then use a spectral sequence given in [4] (or equivalently, an exact couple together with the injectivity of $\left.B_{2 m+1}: H^{2 m+1}\left(A, A^{*}\right) \rightarrow H_{\lambda}^{2 m}(A), m \geq 0\right)$, we obtain $H_{\lambda}^{2 m}(A) \cong \mathbb{C}^{n}, H_{\lambda}^{2 m+1}(A)=0, m \geq 0$, and $H^{\text {even }}(A) \cong \mathbb{C}, H^{\text {odd }}(A)=0$. It is also seen that the generator of $H^{\text {even }}(A) \cong \mathbb{C}$ is coming from the generator of $E_{1}^{0}(A) \cong \mathbb{C}$. 
Let us now come back to the case $A=C[x] /(f)$ with $f(x)=$ $\left(x-\alpha_{1}\right)^{N_{1}} \cdots\left(x-\alpha_{m}\right)^{N_{m}}$. Define $\tau_{1}, \ldots, \tau_{m} \in A^{*}$ by

$$
\tau_{l}(g)=g\left(\alpha_{l}\right), \quad l \leq l \leq m, \quad g \in A .
$$

By using the computation for $\boldsymbol{C}[x] /\left(x^{n}\right)$ together with the direct sum formula in Section 2, we obtain our theorem.

Theorem 6. 1. In the above situation, we have;

(1) $H^{0}\left(A, A^{*}\right) \cong C^{N_{1}+\cdots+N_{m}}$ and $H^{j}\left(A, A^{*}\right) \cong C^{N_{1}+\cdots+N_{m}-m}$ for $j \geq 1$ $\left(N_{1}+\cdots+N_{m}=\operatorname{deg}(f)\right)$.

(2) $H_{\lambda}^{2 j}(A) \cong C^{N_{1}+\cdots+N_{m}}, H_{\lambda}^{2 j+1}(A)=0, j \geq 0$.

(3) $E_{n}^{0}(A) \cong C^{m}, E_{n}^{j}(A)=0, j \geq 1$ for $n \geq 1$. This also means that $H^{\text {even }}(A) \cong \boldsymbol{C}^{m}, H^{\text {odd }}(A)=0$. Furthermore, the generators are given by the evaluation maps $\tau_{l}, 1 \leq l \leq m$, (all of them are zero traces on $A$ ) at each root of $f=0$.

\section{Remark.}

(1) In particular, in the case of $A=C[x] /\left(x^{n}\right), \delta^{(0)}$ pairs nontrivially with the class of identity $[1] \in K_{0}(A)$. This holds also for the case $A=\boldsymbol{C}[x] /(f)$.

(2) Our discussion for $A=\boldsymbol{C}[x] /\left(x^{n}\right)$ works well not only for $\boldsymbol{C}$ but also for any field with characteristic zero, but our discussion for $A=\mathbb{C}[x] /(f)$ works at most for algebraically closed field with characteristic zero, in general.

\section{References}

[1] Burghelea, D., The cyclic homology of the group rings, Preprint Ohio State University (1984).

[2] Cartan H. and Eilenberg, S., Homological algebra, Princeton University Press, 1956.

[ 3 ] Connes, A., Noncommutative differential geometry, Chapter I: The Chern character in $K$ homology, Preprint IHES (1982).

[4] - Noncommutative differential geometry, Chapter II: De Rham homology and noncommutative algebra, Preprint IHES (1983).

[5] Cyclic cohomology and the transverse fundamental class of a foliation, Preprint IHES (1984).

[6] Masuda, T., Cyclic cohomology of the group algebras of free groups, Preprint MSRI (1984).

[7] Cyclic cohomology of polynomial rings, Preprint MSRI (1984).

[8] Loday, J-L. and Quillen, D., Cyclic homology and Lie algebra homology of matrices, Comment. Math. Helv. 59 (1984), 565-591. 
[9] Staffeldt, R., Rational algebraic $K$-theory of certain truncated polynomial rings, Preprint MSRI (1984).

Note added: A similar result was also independently obtained in "A model for cyclic homology and algebraic $K$-theory of 1 -connected topological spaces" by Micheline ViguePoirrier and Dan Burghelea. 
